\title{
Sizing Electric Battery Storage for Electric Racing Motorcycle
}

\author{
A. Rodríguez ${ }^{1}$, J. M. González-González ${ }^{2}$ and J. A. Aguado \\ Department of Electrical Engineering \\ E.T.S.I.I., University of Málaga \\ Ampliación de Teatinos, 29071 Málaga (Spain) \\ e-mail (1): adrianrodriguezdiaz1@ gmail.com \\ e-mail (2): josemanuelgonzalez@uma.es
}

\begin{abstract}
The future perspectives of electric vehicles are getting the attention from major manufacturers. Moreover, they are attracting followers from the field with the most advanced technological development: the competition. In pursuit of victory, competition pushes vehicle components to the limit with a view to optimise the performance of them.
\end{abstract}

The aim of this paper is to develop a sizing algorithm for an energy storage system of a competition motorcycle, in such a way that it can be possible to provide to the motor enough energy to complete the race and the needed power to get the best performance. This algorithm takes into account dynamics and technical characteristics of the analysed motorcycle in order to get an optimal battery storage sizing. Finally, the result of this algorithm is compared with a real competition tests.

\section{Key words}

Sizing, battery, competition, motorcycle, MotoStudent.

\section{Introduction}

Nowadays, electric vehicles are acquiring more importance because of its economics and environmental advantages. One of the most important places for the development of the vehicles technology is the competition, which is considering the electric propulsion as one of the next targets.

The goal of every racing team is to achieve the final victory, which is plenty of difficulties because it is not only necessary to have the fastest vehicle, but also it is essential to have a reliable vehicle which is able to complete each race of a season.

When the competition falls within the scope of electric vehicles, one of the main problems which have to face the engineers of a team is how to guarantee the supply of energy and power at each moment of the race. This difficulty has been widely analysed by the Electrical Engineering Department of the University of Málaga for its participation with the UMA Racing Team in the electric motorcycles race of the MotoStudent competition [1], which encompasses universities from all over the world in the search for the victory. The 2016 edition has been made in the circuit of Motorland, Aragón (Spain).

At present, storage sizing is a popular topic in many fields. Motorcycle dynamic characteristics are important to calculate the optimal battery capacity. These parameters are taken into account by the authors of [2] and [3], which include in their calculations of the necessary energy some of the factors that oppose to the motorcycle movement. However, the precision of their results are affected by the absence of other factors, such as using and average speed instead of the exact speed of the motorcycle at each point of the circuit.

Other authors, for example in [4], have considered for the calculations a speed profile for the analysed circuit. However, these authors have omitted the dynamic characteristics of the motorcycle, reducing the accuracy of the results.

The aim of this paper is, firstly, to analyse the different batteries types available on market and to select one for the project. Secondly, we develop a new algorithm able to consider every dynamic factor of the motorcycle as well as the characteristic of every analysed circuit. Finally, we carry out a comparison between the developed algorithm results, and the energy consumption in a real situation in the circuit of Motorland.

\section{Battery selection}

The selection of batteries for a competition motorcycle is a crucial point in the development of an energy storage system, because there is a need to find a trade-off between enough energy to finish the race, and the available area for the battery. For this reason, it is essential to use batteries with a high grade of specific energy and energy density. 


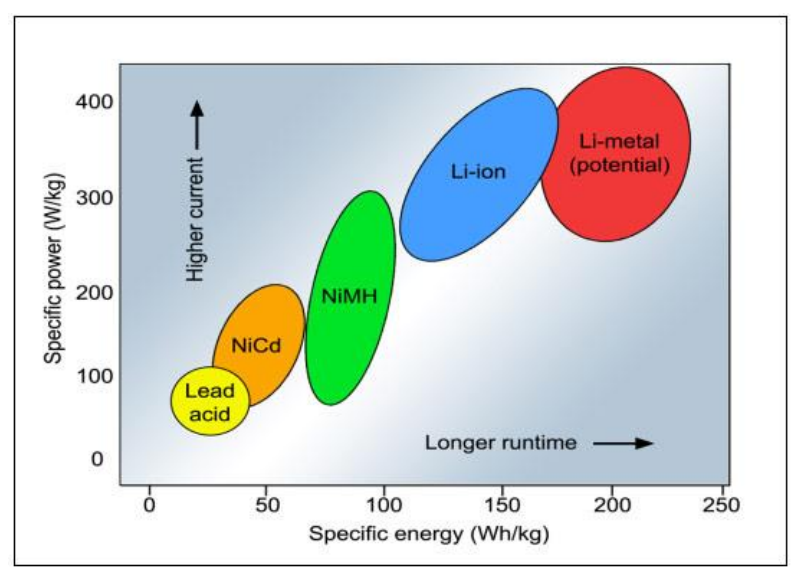

Fig. 1. Comparison between specific power and specific energy of different battery technologies. [5]

Nevertheless, there is another important factor for the election of the battery. Competition motors demand a lot of power to the battery. Consequently, to achieve the design requirements, technologies with high specific power and power density are required.

Figure 1 shows how lithium batteries are ideal for our requirements. This technology is used in some developed competition prototypes; of note among which are Shinden [6] and The Zephyr [7] with Lithium-ion (Li-ion) battery, and MIT eSuperbike [8] and Play and Drive [9] with Lithium-polymer battery (Li-Po).

Table I. - Characteristics of different lithium battery types

\begin{tabular}{|c|c|c|}
\hline Parameter & LiFePO $_{\mathbf{4}}$ & Li-Po \\
\hline Cell Voltage (V) & 3.3 & 3.7 \\
\hline C-Rate & $1-2 \mathrm{C}$ & $20-45 \mathrm{C}$ \\
\hline Specific energy (Wh/kg) & 120 & $130-200$ \\
\hline Specific power (W/kg) & 260 & 315 \\
\hline Efficiency & $90 \%$ & $99 \%$ \\
\hline Number of cycles & $>2000$ & $1000-2000$ \\
\hline Security & Good & Regular \\
\hline Maintenance & Easy & Easy \\
\hline
\end{tabular}

Table I allows to compare different lithium battery types, where it is possible to observe that Li-Po is the technology with the better characteristics for our project because they have high C-Rate values, appropriate for the power requirements of the motor.

\section{Dynamic modelling}

A motorcycle can be modelled as a mass in an incline plane which is submitted to external forces (Figure 2). The electric engine develops a force, $F_{\mathrm{w}}$ in the rear wheel, which is countered by three forces: the aerodynamic drag, $F_{\text {aero }}$, the rolling resistance from the two wheels, $F_{\text {rolling }}$, and the force due to the gravity in the incline plane, $F_{\text {gravity }}$. Consequently, Newton's second law states:

$$
\sum F=M \cdot a=F_{w}-F_{\text {aero }}-F_{\text {rolling }}-F_{\text {gravity }}
$$

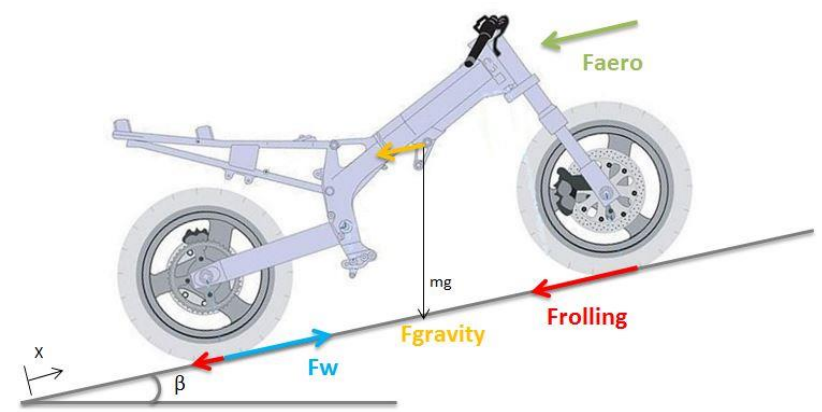

Fig. 2. Dynamic modelling of a motorcycle

Each of these forces can be calculated following the equations shown below, and the parameters reported in Table II, which are related with the motorcycle used by University of Málaga for the Motostudent race.

Aerodynamics drag is the force experimented by the air when it stablishes contact with a vehicle in motion. It depends on the aerodynamics coefficient, frontal area, density of air, and the vehicle speed. It can be calculated as:

$$
F_{\text {aero }}=\frac{1}{2} \cdot C_{d} \cdot A_{f} \cdot \rho \cdot v^{2}
$$

Rolling resistance is the energy loses owing to the friction between the wheel and the road. It depends on the slope of the track, the vehicle mass, gravity, and the rolling resistance coefficient. It can be calculated using the following equation:

$$
F_{\text {rolling }}=C_{r} \cdot M \cdot g \cdot \cos (\beta)
$$

The force exerted in the vehicle by gravity is influenced by the angle of the track, which develops an opposing or in favour force to the movement. It can be obtained as:

$$
F_{\text {gravity }}=M \cdot g \cdot \sin (\beta)
$$

Finally, the force on the rear wheel, $\mathrm{F}_{\mathrm{w}}$, is obtained from the ratio between the torque provided by the electric engine through the gear box, chain and sprockets (each of them with its efficiency), and the wheel radius. It is defined in Equation 5.

$$
F_{w}=\frac{\tau_{\text {motor }} \cdot z \cdot \eta}{r_{w}}
$$

Table II. - Parameters used in dynamic modelling

\begin{tabular}{|c|c|c|c|}
\hline Parameter & Variable & Value & Unit \\
\hline Frontal area & $\mathrm{A}$ & 0.70 & $\mathrm{~m}^{2}$ \\
\hline Aerodynamic coefficient & $\mathrm{C}_{\mathrm{d}}$ & 0.42 & - \\
\hline Rolling resistance coefficient & $\mathrm{C}_{\mathrm{r}}$ & 0.02 & - \\
\hline Mass vehicle + rider & $\mathrm{M}$ & 175 & $\mathrm{~kg}$ \\
\hline Density of air & $\rho$ & 1.23 & $\mathrm{Kg} / \mathrm{m}^{3}$ \\
\hline Gear ratio & $\mathrm{z}$ & 4.64 & - \\
\hline Tire radius & $\mathrm{r}_{\mathrm{w}}$ & 0.30 & $\mathrm{~m}$ \\
\hline Efficiency drivetrain & $\eta_{\text {drivertain }}$ & 0.90 & - \\
\hline
\end{tabular}




\section{Battery sizing}

The process described above allows us to obtain the motorcycle acceleration force as a function of speed $(v)$. To determine these velocities at each point of the track, it used the Equation (6). In this equation, it is stablished the parameter space $(s)$ as a constant equal the unity, which means it is calculated the speed each meter of the track, obtaining the upper limit, which corresponds to the speed value at the end of the meter. The lower limit represents the speed at the beginning of the meter. This velocity is obtained from a Moto3 speed profile telemetry data in the Motorland circuit, which can be assumed equal in curves.

$$
s=M \cdot \int_{v 0}^{v f} \frac{v}{F_{\text {accel }}(v)} d v
$$

As a result of the values obtained by Equation (6), the team acquires the estimated speed value at each point of the track, creating a reference speed profile. It is shown in the Figure 3.

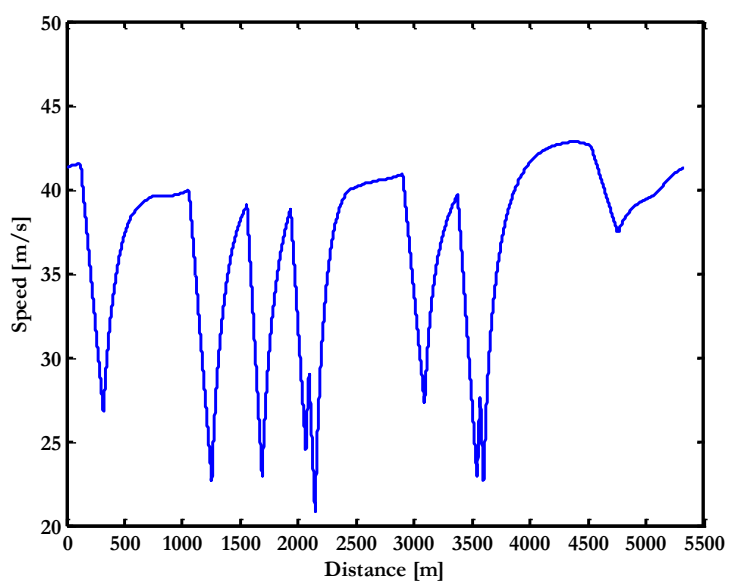

Fig. 3. Speed Profile of the UMA Racing Team electric motorcycle throughout a reference lap.

Furthermore, to calculate this speed profile, notice the necessity to use an algorithm to implement the equations described in previous sections, due to the complexity and the large number of iterations required to solve them (MATLAB 12 is used in this case). It has been developed in a MATLAB script based in the diagram shown in Figure 4

Once the reference speed profile is known (Figure 3), and applying Equation (7), we can know how much power the electric engine demands at each instant of time and, applying Equation (8), how much energy (kWh) the battery needs to supply it (taking the efficiencies of controller and electric engine as 0.95 [10] and 0.90 , respectively).

$$
\begin{gathered}
P_{\text {engine }}=\tau_{\text {engine }} \cdot \omega_{\text {engine }} \\
E_{\text {battery }}=\frac{P_{\text {engine }} \cdot t}{1000 \cdot 3600 \cdot \eta_{\text {controller }} \cdot \eta_{\text {engine }}}
\end{gathered}
$$

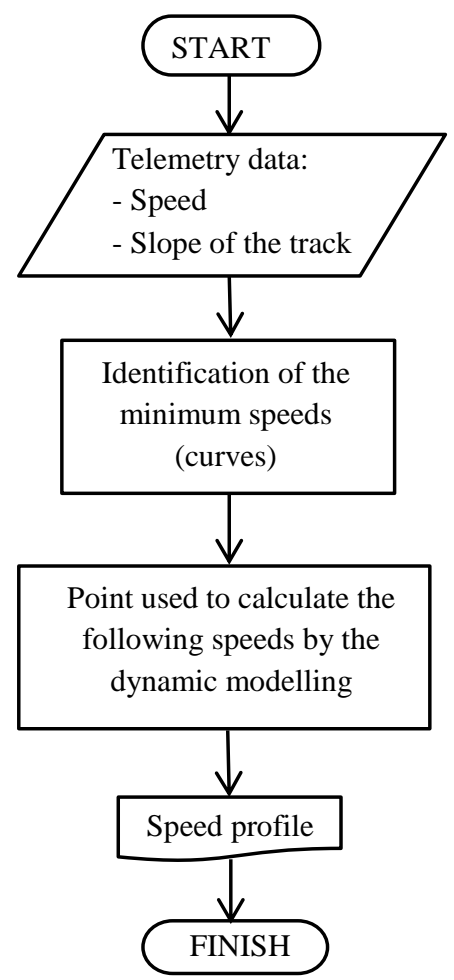

Fig. 4. Proposed algorithm to calculate the reference speed profile.

In Equation (7) and (8), it is combined two types of data in order to obtain the necessary energy to finish a lap. On the one hand, we have the estimated speed data of the motorcycle. These speed data are converted to revolutions using the gear ratio. On the other hand, we have the torque of the electric engine vs revolutions, which is represented by the red curve in the Figure 5.

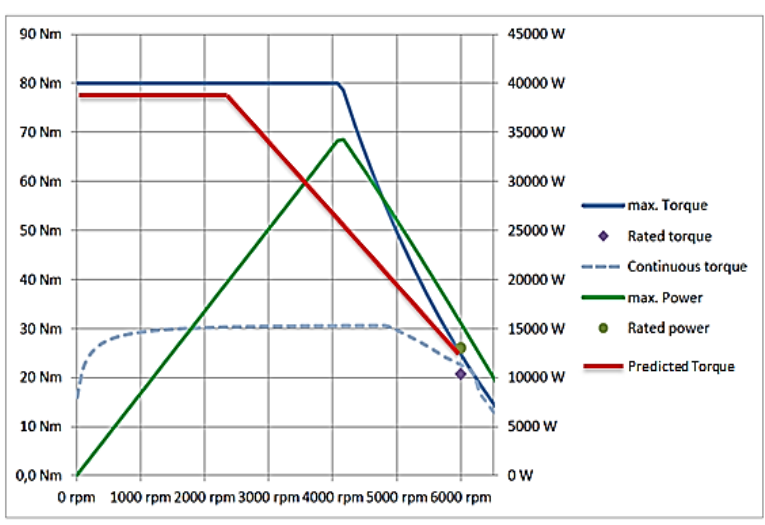

Fig. 5. Electric motor characteristics curve [10].

\section{Results}

The main objective of this work is to develop an algorithm to analyse the circuit where a race takes part and determine the necessary energy to finish it. This section shows the results obtained for the Motorland circuit with two different methods: a method without energy re-generation, and other one with energy regeneration using a regenerative braking system. Finally, 
the real race results are shown in order to make a comparison with analytical results.

\section{A. Battery sizing without energy re-generation}

Once we obtain the estimated speed profile for the motorcycle, the algorithm can calculate as the power demanded at each moment as the energy consumed throughout the race.

Figure 6 shows the reference profile of the power demanded by the electrical motor during a lap of the race.

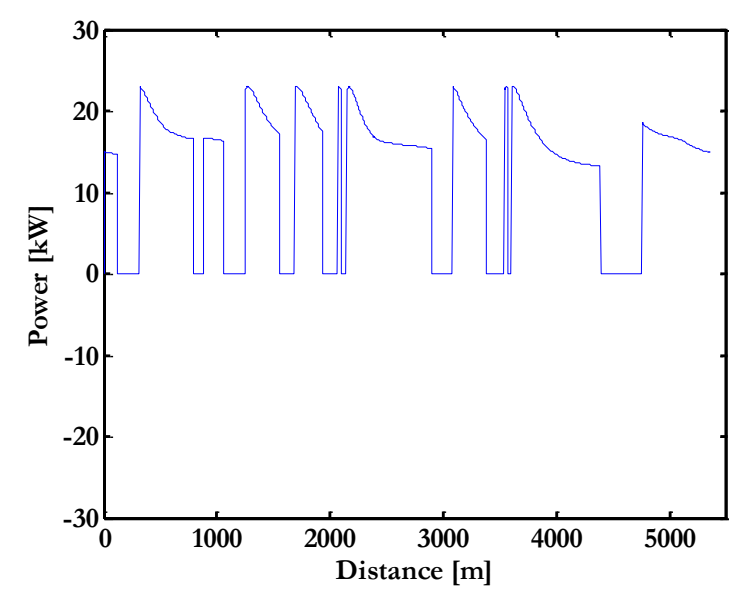

Fig. 6. Power demanded throughout a reference lap.

The power profile shown above is used by the algorithm to calculate the total energy needed to complete the race. According to competition rules, the battery has to be capable to supply energy during the 5 laps of the race; moreover, it is necessary to complete the recognition lap and the last lap to return to the pit boxes. The telemetry data from other motorcycles show that the energy consumed in these two laps is over $70 \%$ of a regular lap.

According to the previous factors, the efficiency of the system, and the power profile, the total energy required to complete the race is $4.56 \mathrm{kWh}$.

\section{B. Battery sizing with energy re-generation}

The reduction of the size and weight of the storage system is a key factor in the development of an electric motorcycle because it affects directly to the speed, manoeuvrability and power consumption.

One way to achieve this objective is to implement a regenerative braking system in the electric motorcycle. This system not only allows to flow the energy from the battery to the electric motor, but also from the electric motor to the battery (Figure 7), recovering the energy of each braking in order to charge the battery.

The main problem of a regenerative braking system in a motorcycle is the quantity of energy that can be generated by the electric motor. In a motorcycle, the braking action is distributed $60 \%$ to the front wheel, and $40 \%$ to the rear wheel, approximately. The traction system is constituted by the rear wheel so the energy generated is only $40 \%$.

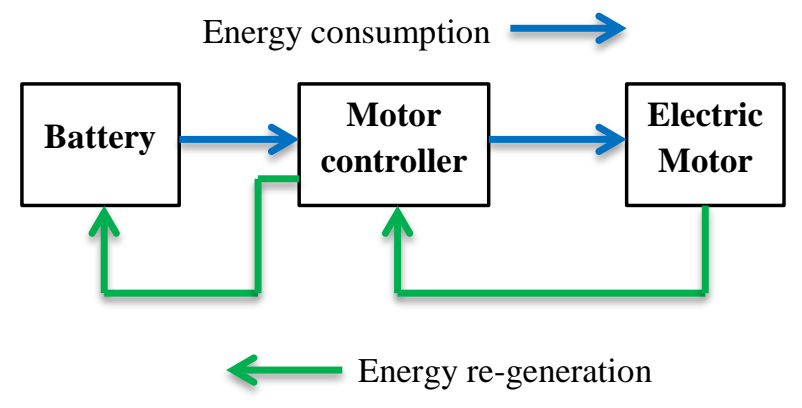

Fig. 7. Energy flow when electric motor consumes energy and when it provides energy to the battery.

Moreover, the electric engine does not work the same way operating as motor and as generator, being the efficiency to work as a generator $80 \%$. Thus, the total energy is estimated at $300 \mathrm{Wh}$. The Figure 8 shows the power demanded (positive power) and generated (negative power) during a lap.

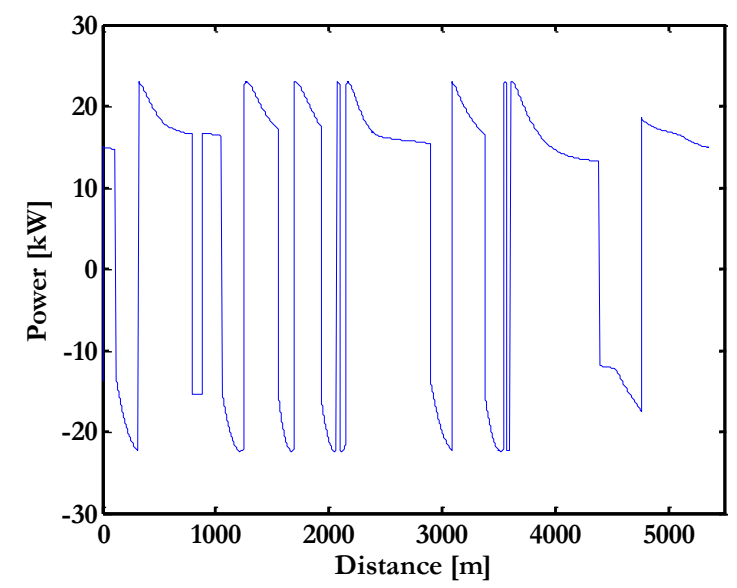

Fig. 8. Power demanded and generated with the regenerative braking system throughout a lap.

In spite of the low energy recovered in this case, this system could be interesting in competitions with a large number of laps.

Finally, a solution without a regenerative braking system has been adopted in the motorcycle. Due to safety factors, it has been designed a battery of $4.80 \mathrm{kWh}(50$ $\mathrm{Ah})$ instead of $4.56 \mathrm{kWh}(47.47 \mathrm{Ah})$ as the algorithm have calculated.

\section{Race results}

The University of Málaga motorcycle participated in the final race during the contest in Motorland (Figure 9) finishing $5^{\text {th }}$ out of 20 . Analysing the real results of the race, the team has obtained the energy consumed during the race which has been $4.53 \mathrm{kWh}(47.14 \mathrm{Ah})$.

According to this result, it concludes the algorithm developed, and the data used to solve the equations, have provided an accurate result, being $0.03 \mathrm{kWh}$ the difference between analytics values and real values. However, the excess energy in the battery was higher due to the safety factor applied. 


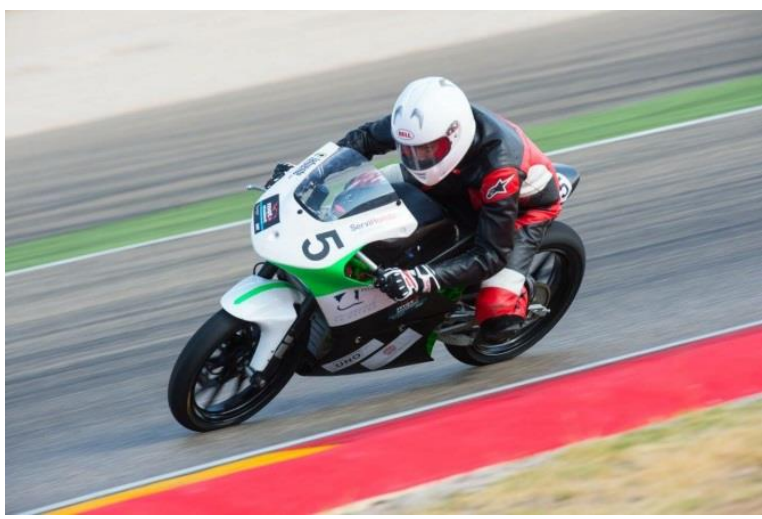

Fig. 9. The motorcycle during the MotoStudent race.

\section{Conclusions}

This paper has described the methodology for sizing a Lithium-polymer battery to be used as energy storage for the electrical propulsion of a MotoStudent electric race motorcycle. Batteries based in lithium have been chosen due to the trade-off between energy and power, concluding Lithium-Polymer batteries are those which present the best characteristics for a racing motorcycle because they have high C-Rate values needed to supply the high power requirements.

The battery sizing has been performed using the algorithm developed in this paper, being able to obtain a solution from telemetry data of the circuit and motorcycle characteristics.

The results obtained show the precision of the developed algorithm used to size the battery. However, it can be observed the advisable use of a safety coefficient to avoid variations between the analytical results and the real ones.

Finally, after analysing a solution with and without a regenerative energy system, the former has been selected because of the MotoStudent competition has a low number of laps.

\section{References}

[1] MotoStudent 2015-2016 Rules and Regulations (2016, October 10) [Online]. Available:

http://www.motostudent.com/archivos/RegulationsMS 1516EN.pdf.

[2] L. Rodgers, R. Gogoana and T. German, "Designing an electric motorcycle for the Isle of Man TT Zero race, and how electric vehicle racing could be used to spur innovation", EVS International Battery, Hybrid and Fuel Cell Electric Symposium, Los Ángeles, California, 2012, pp. 1-12.

[3] F. A. Nugraha, A. Purwadi, Y. Haroen and N. Heryana, "The calculation of electric motor and Lithium battery capacity on Cikal Cakrawala ITB electric car", IEEE Conference on Power Engineering and Renewable Energy, Bali (2012)

[4] F. Baaronti, D. Calderini, G. Caposciutti and A. Gassani, "The calculation of electric motor and Lithium battery capacity on Cikal Cakrawala ITB electric car", IEEE International Symposium on Industrial Electronics, Istambul (2014)

[5] Comparison between specific power and specific energy of different battery technologies (2016, October 3) [Online] Available: http://www.batteryuniversity.com

[6] Autoblog: Mugen debuts new Shinden electric motorcycle for TT Zero race (2015, October 9) [Online]. Available: http://www.autoblog.com/2014/04/08/mugen-debuts-newshinden-electricmotorcycle-for-tt-zero-race/

[7] Zeus official website team (2016, October 3) [Online]. Available: http://www.suclubs.orgsync.com/org/teamzeus/Zephyr

[8] MIT News: Extreme electric racing (2015, October 9) [Online]. Available: http://www.news.mit.edu/2011/esuperbikeisle-of-man$\underline{0610}$

[9] Oficial website of Play and Drive (2015, October 9) [Online]. Available: http://www.playanddrive.com/ prototipos/motostudentelectric/

[10] Datasheet Heinzmann® PMS 150, ID 490, Heinzmann, Selva Negra, Germany, 2015. 